\title{
SELECTIVE CINE-ANGIOCARDIOGRAPHY WITH IMAGE INTENSIFICATION
}

\author{
BY \\ HAMISH WATSON, C. PICKARD, K. G. LOWE, AND IAN HILL \\ From the Departments of Medicine and Radiology, University of St. Andrews \\ Received February 12, 1958
}

There has been steady progress in angiocardiographic technique since Castellanos et al. (1937) showed that satisfactory opacification of the heart chambers was possible in children, and Robb and Steinberg (1938) confirmed that equally successful results could be obtained in adults. Though the earliest attempts to visualize the interior of the heart had been unsuccessful, it is interesting to note that in these the contrast medium had been introduced directly into the heart (Forssmann, 1931; Pérez Ara, 1931; and Moniz et al., 1931). Most of the subsequent work was done using intravenous injection, and it was not until 1946 that a reliable method of direct intracardiac angiocardiography was described by Chavez et al. (1947). Since then much thought has been given to methods for rapid and safe intracardiac injection (Jönsson et al., 1949; Dorbecker et al., 1954; Gidlund, 1956; and Rodríguez-Alvarez and de Rodríguez, 1957) and, though they are not yet perfect, many of their earlier dangers and disadvantages have been overcome.

The introduction of image intensification by Teves and Feddema (1953 and 1955) started a new era in diagnostic radiology and the combination of the intensifier with a cine camera has provided an

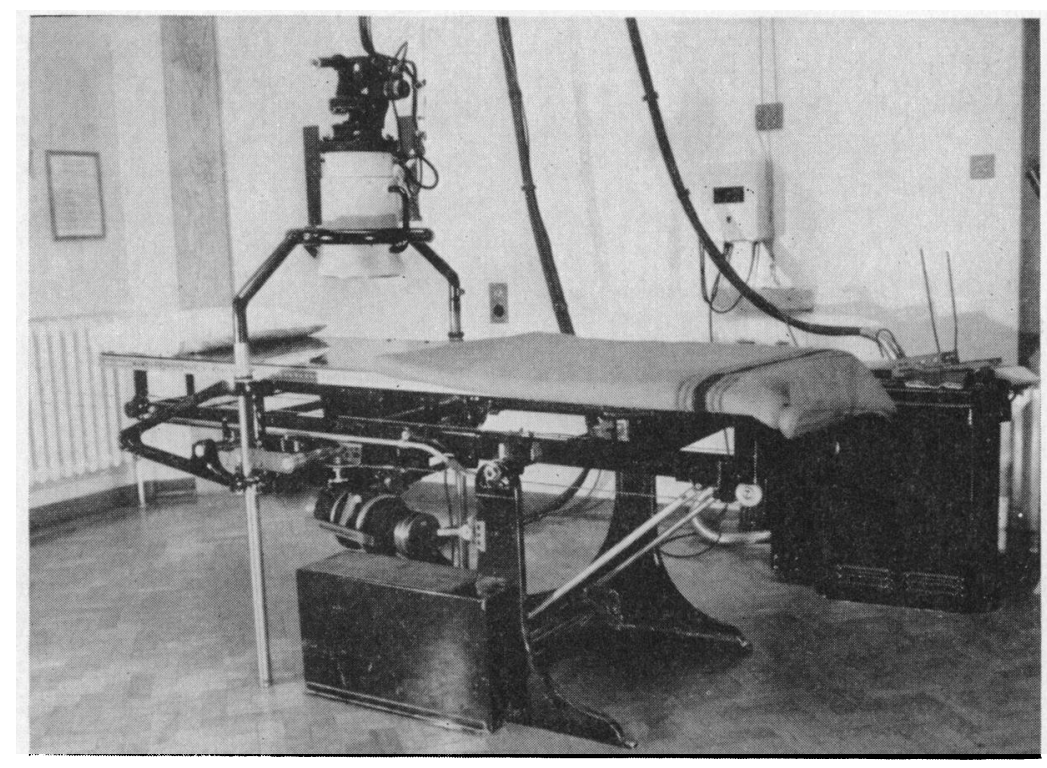

FIG. 1.-This shows the general arrangement of the tilting table with a mobile top, and the radiological apparatus. 459 
instrument that is particularly well suited to the field of angiocardiography (Lind and Wegelius, 1955; Astley, 1955 and 1956; and Stauffer et al., 1955). When it is used with a good technique of selective injection, the films made can be studied either in motion, at normal or slow speeds, or projected as serial stills, and this results not only in more accurate diagnosis but allows detailed study of the actual intracardiac circulation. In addition the comparatively small radiation rates used (Feddema, 1953; and Lind et al., 1955) mean much greater safety for both patients and staff-a factor now rightly considered of the utmost importance.

\section{EQUiPMENT AND MeTHODS}

The installation is illustrated in Fig. 1 and some results in Fig. 2, 3, and 4. It consists of a tilting table with a mobile top. The undercouch tube is a Super Dynamax with a $1 \mathrm{~mm}$. focal spot, and the focus is 24 inches $(60 \mathrm{~cm}$.) from the skin surface. The operator controls the beam delineation by a double diaphragm which affords full protection. Power is supplied to the tube from a Watson cine-generator and, as the H.T. cables are long, no further stabilization is required with low continuous tube currents. The explorator has been replaced by a Philips 5 -inch $(12.5 \mathrm{~cm}$.) intensifier which can be used with a fluorescent tunnel, a direct lens system, or a 35-mm. cine camera. Standard fluorescent screen examination is also possible over a 12-inch $(30-\mathrm{cm}$.) square area, and spot films can be taken if required.

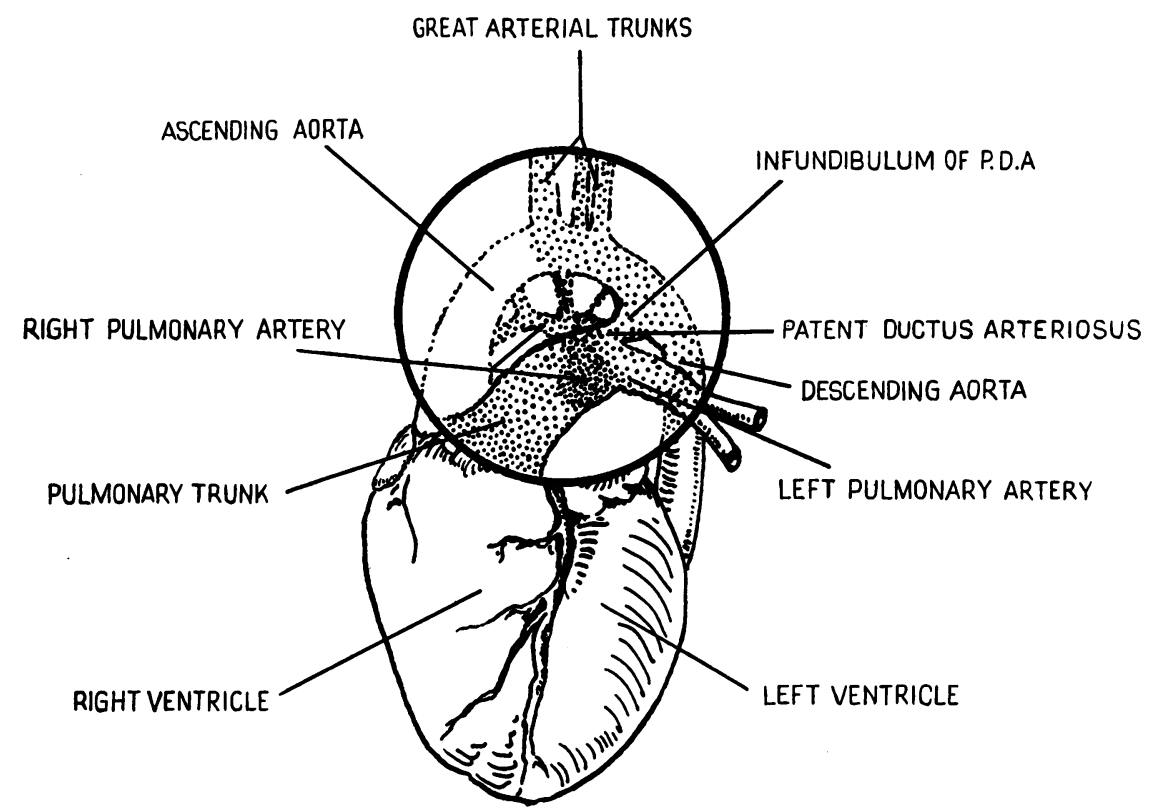

FIG. 2.-The relationship of the intensifier field to the heart and great vessels (left anterior oblique view).

FIG. 3 and 4 illustrate two sets of twelve consecutive pictures taken at 50 frames a second, from a selective cineangiocardiogram done on an 8-year-old girl, who presented as a case cf Eisenmenger's syndrome (Wood, 1952). The investigation was carried out after clinical examination and cardiac catheterization had failed to differentiate between a ventricular septal defect and patency of the ductus arteriosus.

FIG. 3.-In pictures 1-5 the initial opacification of the patent ductus arteriosus is seen developing and its infundibulum (Steinberg et al., 1943; Jönsson and Saltzman, 1952) is clearly defined. The contrast medium outlines the descending aorta (6 and 7), and thereafter retrograde opacification of the first part of the descending aorta and the aortic arch takes place (8-12). See page 461, opposite.

FIG. 4.-The first picture, at the end of diastole, shows that the innominate artery and the distal half of the aortic arch are opacified. In the second picture systole begins and the non-opaque blood from the left ventricle "washes out" the contrast medium down to the level of ductal entry (2-7); with diastole the retrograde opacification begins again and progresses upwards (8-12). See page 463. 


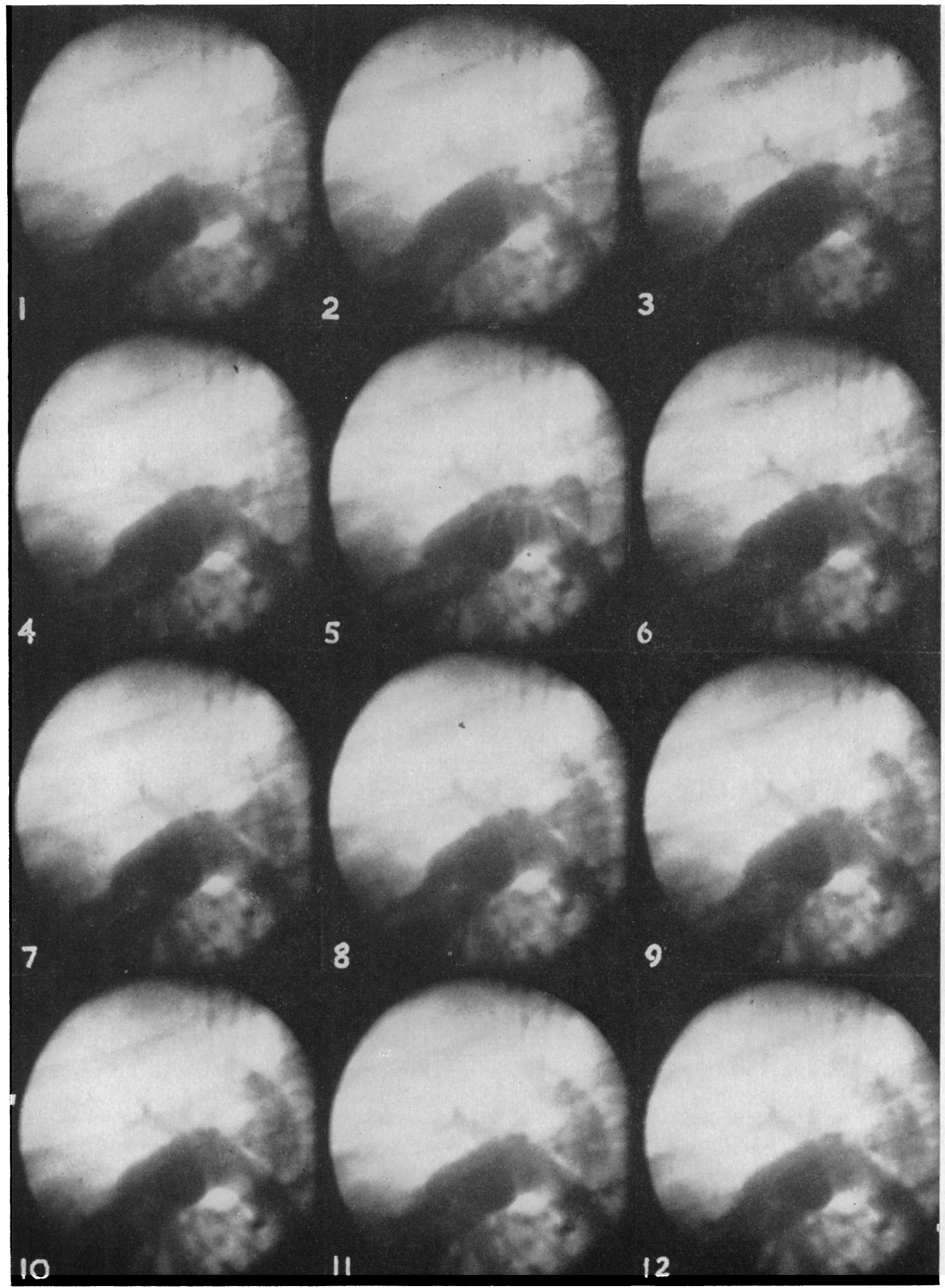

FIG. 3. 
The control of the exposure for cine-radiography is now standard. A micro-voltmeter is in circuit and arbitrary figures have been established that correlate the size and thickness of patient, the speed of the camera, and the radiographic output from the tube. This system has been satisfactory for all the speeds so far used. At lower speeds radiographic factors of the order of $65 \mathrm{KV}$ and $1 \mathrm{MA}$ are utilized, but at higher speeds, $100 \mathrm{KV}$ and $5 \mathrm{MA}$ are required. A stationary grid has been found an advantage and the filtration of the tube varies from 0.5 to $3 \mathrm{~mm}$. depending upon the $\mathrm{KV}$ applied. As the period during which cine-radiography is in operation does not usually exceed 12 seconds, it has not been found necessary to involve complicated switching devices. During the preparation of the installation, much detailed work was carried out on the photographic aspects of this technique, and after experimenting with a variety of methods, HPX or Scopix G.35-mm. film, processed under standard conditions with standard solutions, has been found satisfactory for most cases, and the master film is available for preliminary examination within a few hours.

There is no doubt that at present the main disadvantage of image intensification is the small size of the screen, and like others we have found that this rather limits its usefulness. But as the investigation of congenital heart disease is largely confined to children, the 5 -inch $(12 \cdot 5-\mathrm{cm}$.) field is less restrictive than would at first sight be imagined. It does, however, mean that, as we can at present only film in one plane, the greatest care must be taken with the positioning of the patient if the maximum information is to be obtained. In our preliminary studies we have used variations of the left anterior oblique view, depending on the nature of the lesions present, and have found that this gives good visualization of the heart chambers and great vessels. In infants and young children, where the size of the screen is no problem, this represents a good compromise in the absence of the usual biplane photography and gives satisfactory results. When it is not possible to get the whole heart on the screen, the field is centred on the particular area that will give most help in the diagnosis and future surgical treatment.

If in addition to diagnosis and pre-surgical evaluation, the intracardiac circulation is to be studied, it is essential that the injection be truly selective. To make certain of this is by no means easy because the action of the heart, the rotation of the patient into the desired position, and the force of the injection itself tend to produce movement of the catheter tip, and may cause it either to change its position within the cavity or to move out of it altogether. If, for example, the right ventricular outflow tract and pulmonary valve are to be studied, the contrast medium is injected into the lower part of the right ventricle with the intention that ventricular contraction will push it up into the infundibulum. If the catheter moves up or points up into the infundibulum during the injection, the result is a pulmonary arteriogram and neither the filling mechanism nor the anatomy of the outflow tract will be defined. On the other hand should the force of the injection push the tip of the catheter back into the right atrium, only the initial opacification takes place in the ventricle, and very quickly the unwanted right atrial shadow obscures the detail of the outflow tract and pulmonary valve.

The size of the catheter used varies and is not necessarily the largest one possible. When selecting it, the size of the heart as well as the size and age of the patient have to be considered carefully and in the light of previous experience, if good pictures are to be obtained. For babies a No. 5 catheter is used and the injection given as rapidly as possible, but with larger sizes in older children the force of the injection is varied to suit the problem that is being investigated, and the tip of the catheter is shaped and fixed to match the size of the heart and the site of injection in each case. A leg vein is used for the catheterization for two reasons: firstly because in babies and very small children it is easier, and secondly because we have found that if an arm vein is used and films are taken in oblique positions, the catheter may obscure structures to be visualized. When the catheter is in the desired position, it must be securely anchored to the skin of the limb if the force of the injection is not to cause it to change position, and the use of catheters with lateral holes near the tip does much to reduce recoil during the injection.

Not only the choice of the site of the injection, but also the means used to ensure that the contrast substance is in fact delivered there, have an important effect on the value of what is eventually 


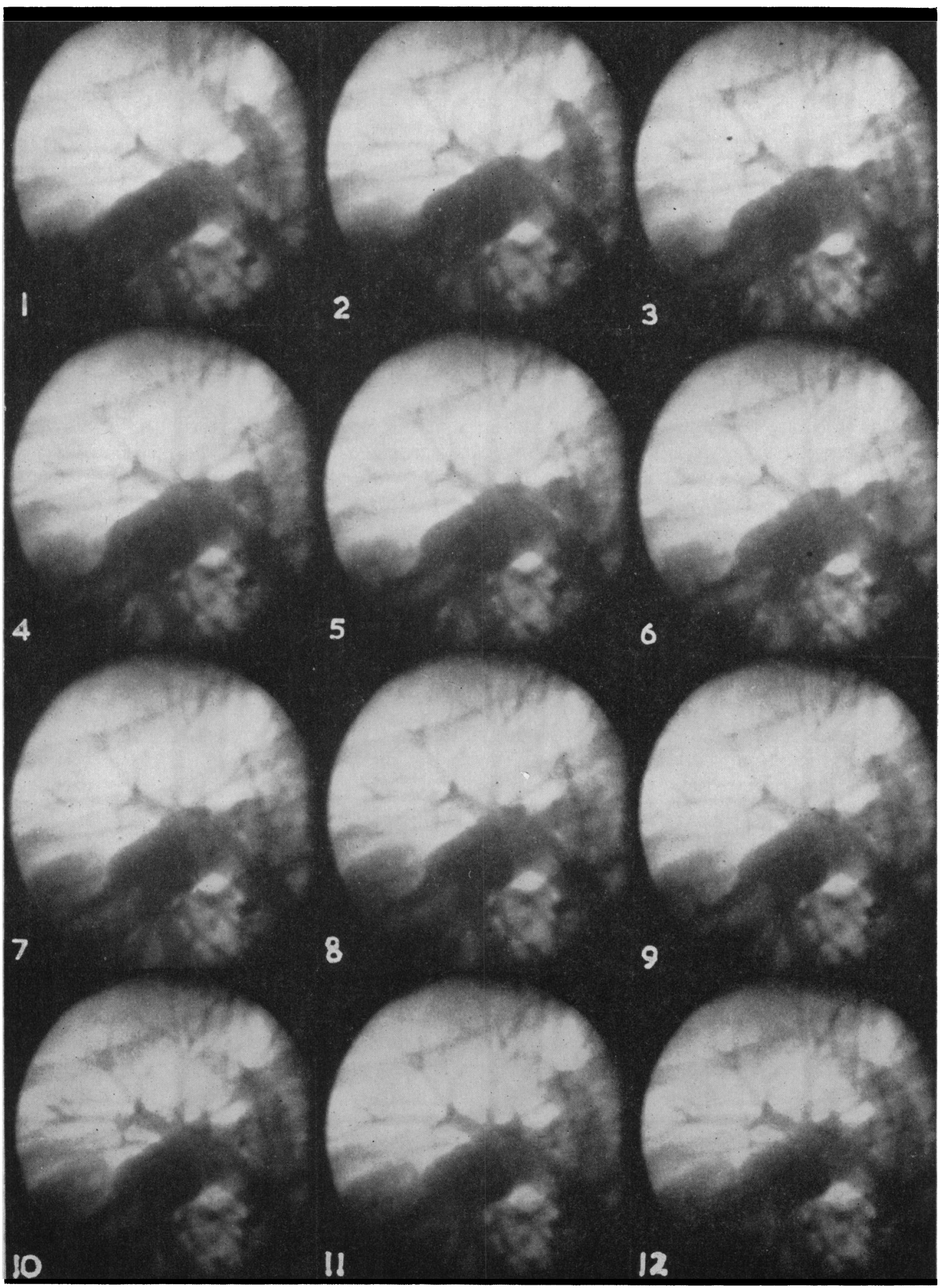

Fig. 4. 
seen on the cinematograph screen. As the field to be photographed can be seen through a lens or tunnel in full lighting, it is a simple matter to check the position of the patient and the site of the catheter tip right up to the moment of injection.

When angiocardiograms are studied in motion, opacification is much more obvious than it is on still pictures, and can be seen where, on stills, there would be some doubt as to whether or not faint delineation is present. With our usual technique ultra-rapid injection is not required, and the aim is to produce adequate opacification over several cardiac cycles. However, since at rapid film speeds faint delineation is fairly obvious, we have been trying two or more smaller injections in different planes, though still keeping the total dose of contrast medium within normal limits. For this it is essential that the small volume of contrast substance enters the heart as a bolus, and the object is to be sure of catching at least one complete cardiac cycle showing the initial opacification. Though there are obvious drawbacks we hope that some such modification in technique will prove satisfactory for selected cases and that in this way still more information will be obtained.

The problem of correlating angiocardiographic films with events in the cardiac cycle is one that has in the past received surprisingly little attention, perhaps because it is not vitally important in many cases with slower film speeds. With cine-photography accurate timing is of first importance if the intracardiac circulation is to be studied in detail. A simple device that produces automatic exposure marking on a synchronous electrocardiogram (Lind et al., 1954) has been successfully modified for this purpose and the time relationship of single pictures can be determined. If in addition the duration of the injection is shown on the recording paper, an exact correlation between electrocardiogram, X-ray picture, and injection of contrast medium is produced. This time relationship can be further checked, if by means of a simple circuit a given electrocardiographic signal, say the top of the $\mathrm{R}$ wave, produces a light spot on the film.

One of the major difficulties encountered has been in the presentation of the results for analysis. This has been tackled in various ways (Campeti et al., 1955; Greenwood, 1956) but the general principle that we have now adopted is to have the master film, approximately $80-100 \mathrm{ft}$., processed, examined for possible faults, and then sent away for professional copying. As it is difficult to project $35-\mathrm{mm}$. film in this country because of the existing safety regulations, the prints are reduced to $16 \mathrm{~mm}$. and there is little or no loss of quality or contrast in the process. We have found the definition on the $35-\mathrm{mm}$. film quite satisfactory, and because we think analysis is easier when the contrast medium appears black, a positive $16-\mathrm{mm}$. film is made from the original $35-\mathrm{mm}$. negative. Detailed examination is not carried out until the prints are available, and though this means a certain amount of delay, the master film is protected from wear and tear and filed for future reference.

It takes time to adapt to this new method of presentation, and the eye accustomed to conventional angiocardiography finds that the speed of events is a little confusing. When running the film through as serial stills it has to be constantly borne in mind that the time interval between pictures is very small: otherwise, a false impression of persistent opacification in this or that chamber or vessel will be created. We are convinced that slow motion projection is essential for detailed studies, but at present this is not easy to achieve. The maximum camera speed commonly in use is about 50 frames a second, and is too slow for a genuine slow motion effect. This can, however, be simulated by using skip-frame optical projection in printing, which though very effective is also very expensive. The aim must therefore be to increase the camera speeds so that slow motion projection is possible, and with this in mind we have now produced satisfactory films with more than 70 frames a second. It has been suggested that image persistence may arise at faster speeds, and though this may well be a problem in the future, we have not been troubled by it so far.

\section{Discussion}

Angiocardiography, while presenting a factual record for analysis, is very much subject to the personal factor in interpretation. Nowhere perhaps is this more true than in the study of the opacification of the right ventricular outflow tract in pulmonary stenosis: here the greatest difficulty 
may be experienced in distinguishing between the normal infundibulum in varying stages of contraction and infundibular stenosis. The healthy outflow tract is funnel-shaped and of much greater diameter than the adjacent pulmonary trunk, but in our experience this fully dilated phase is a very momentary event, and in a child with a fairly rapid heart rate the chances of catching it on single films are very small. The main problem facing those interested in angiocardiography has therefore been to get increased film speeds without raising the already high radiation rates. The use of image intensification and cine-photography not only helps to solve this problem, but makes it possible to take five times as many pictures per second as the fastest conventional machines with a considerable reduction in the dose of X-rays received by the patient. Though the methods of its application are still in a developmental stage it is already obvious that this is an advance in diagnostic technique. It is, for example, always possible at these film speeds to see the right ventricular outflow tract fully dilated and to decide whether narrowing is present or not, and this kind of detailed study of the intracardiac circulation in patients with congenital heart disease will lead to a much clearer understanding of the abnormal mechanisms involved (Fig. 2, 3, and 4).

\section{SUMMARY}

Selective cine-angiocardiography using a Philips image intensifier is described, and the technical problems of production and presentation of the films for analysis are discussed. The future possibilities of this method in the investigation of congenital heart disease are emphasized.

\section{REFERENCES}

Astley, R. (1955). Brit. J. Radiol., 28, 221.

(1956). Brit. J. Radiol., 29, 556.

Campeti, F., Gramiak, R., Watson, J. S., Ramsey, G. H., and Weinberg, S. (1955). Circulation, $12,199$.

Castellanos, A., Pereira, R., and Garcia, A. (1937). Arch. Soc. estudios clin. Habaña, 31, 523.

Chavez, I., Dorbecker, N., and Celis, A. (1947). Amer. Heart J., 33, 560.

Dorbecker, N., Rodríguez-Alvarez, A., and Volnie, B. (1954). II Congreso de Cardiologiá SIBIC-Acapulco, Mexico.

Feddema, J. (1953). Annual Congress, Brit. Institute of Radiology.

- (1955). Brit. J. Radiol., 28, 217.

Forssmann, W. (1931). Münch. med. Wchnschr., 78, 489.

Gidlund, A. (1956). Acta Radiol., Supplement, 130.

Greenwood, F. G. (1956). Brit. J. Radiol., 29, 544.

Jönsson, G., Brodén, B., and Karnell, J. (1949). Acta Radiol., 32, 486.

- and Saltzman, G. F. (1952). Acta Radiol., 37, 445.

Lind, J., Wegelius, C., and Lichtenstein, H. (1954). Circulation, 10, 195.

- - (1955). Nord. Med., 22, 914.

,-- , and Boesen, I. (1955). Henry Ford Symposium on Cardiovascular Surgery, Detroit.

Moniz, E., de Carvalho, L., and Lima, A. (1931). Presse méd., 39, 996.

Pérez Ara, A. (1931). Rev. méd. circ. Habaña, 36, 7.

Robb, G. P., and Steinberg, I. (1938). J. clin. Invest., 17, 507.

Rodríguez-Alvarez, A., and de Rodríguez, G. M. (1957). Amer. Heart J., 53, 481.

Stauffer, H. M., Morton, J., Oppenheimer, M. J., Stewart, G. H., and Blackstone, A. W. (1955). Radiology, $65,784$.

Steinberg, M. F., Grishman, A., and Sussman, M. L. (1943). Amer. J. Roentgenol., 50, 306.

Teves, M. C. (1953). Annual Congress, British Institute of Radiology.

- (1955). Brit. J. Radiol., 28, 216.

Wood, P. (1952). Brit. med. Bull., 8, 348. 